\title{
RELACIÓN ESPACIAL ENTRE PROPIEDADES DE SUELOS Y ESTRÉS HÍDRICO EN CAÑA DE AZÚCAR EN LA HACIENDA CHURIMAL- ROLDANILLO-VALLE DEL CAUCA, COLOMBIA
}

\author{
Julio Cesar Díaz Serna ${ }^{1} \varangle$
}

\begin{abstract}
1 ingeniero Agrónomo, Maestría en Sistemas de información Geográfica. agronomi.10@hotmail. com

PALABRAS CLAVES: Interpolación, fenología de la planta, requerimiento hídrico, manejo de riegos y manejo de suelos
\end{abstract}

\begin{abstract}
RESUMEN
El cambio climático es un problema importante en la agricultura, y es necesario tomar medidas en diferentes aspectos para reducir sus efectos negativos (altas temperaturas, alto brillo solar, alta evaporación). Como consecuencia del cambio climático, la producción agrícola se verá afectada en un alto porcentaje, específicamente en la reducción del área disponible y en una menor productividad. En los últimos años, el estrés relacionado con el agua ha sido uno de los factores que ha influido negativamente en las actividades agrícolas en la finca Churimal, Roldanillo en el Departamento del Valle del Cauca en Colombia. La metodología consistió en: i) georreferenciación de los puntos ubicados en el área plantada 1, ii) análisis físico de las muestras de suelo en el laboratorio y, iii) la sistematización de los datos obtenidos en campo, mediante las herramientas de ArcGIS. Los métodos de interpolación espacial fueron utilizados en este estudio, apoyado por el software ArcGIS (Arcmap) y su herramienta de interpolación IDW, la cual es práctica, flexible y muestra como es la distribución espacial y la cuantificación de cada variable analizada en ambas capas de suelo y subsuelo. Además, se analizó la evolución histórica de la precipitación anual (2011 a 2016), junto con las propiedades físicas del suelo en el área de estudio. Con base en los requerimientos de agua del cultivo de caña de azúcar, se desarrolló un enfoque teórico de la aplicación de riego en el cultivo de la caña de azúcar.

Se obtuvieron resultados que muestran las relaciones entre la planta, el clima y las características del suelo. Por lo tanto, se presentaron mapas que caracterizan las propiedades físicas del suelo en el área plantada. Se analizaron y graficaron los datos históricos de precipitación entre los años 2011 y 2016 y finalmente se determinaron las necesidades en agua del cultivo de caña de azúcar relacionado con el agua existente de la precipitación. Por lo anterior se concluyó que existe déficit hídrico en el cultivo de caña de azúcar en alto periodo de su fenología.
\end{abstract}

\section{SPACE RELATIONSHIP BETWEEN SOIL PROPERTIES AND WATER STRESS IN CAÑA DE AZÚCAR IN THE HACIENDA CHURIMAL-ROLDANILLO-VALLE DEL CAUCA, COLOMBIA}

\section{KEYWORDS}

Interpolation, plant phenology, water requirement, irrigation management and soil management.

\section{ABSTRACT}

Climate change is an important problem in agriculture, and it is necessary to take actions in different aspects in order to reduce its negative effects such as high temperatures, solar brightness and evaporation. Because of climate change, agricultural production will be affected in a high percentage, specifically in the reduction of available area and lower productivity. In the last years, water related stress has been one of the factors that has negatively influenced agricultural activities in the Churimal Roldanillo farm, Valle del Cauca department in Colombia.

The methodology consisted on: i) georeferencing the located points in planted area 1, ii) physical analysis of soil samples in laboratory and, iii) the systematization of field work data using the ArcGIS tools. Spatial interpolation methods were used in this study, supported by the ArcGIS (Armap) software and its IDW interpolation tool, which is practical and flexible. It shows spatial distribution and quantification of each analyzed variable in both top-soil and sub-soil layers. In addition, annual precipitation historical evolution (2011 to 2016) was analyzed with physical soil properties in the study area. Based on water requirement of sugarcane crop, a theoretical approach of irrigation application in the cultivation of sugarcane was developed. Results showed relations between plant, climate and soil characteristics. Thus, a serie of maps was presented characterizing soil physical properties distribution in the planted area. Historical precipitation data between the years 2011 to 2016 were analyzed and graphed and finally sugar cane cultivation water requirement was analyzed in relation to existing water from precipitation. Therefore, it was concluded that there is a water deficit for sugar cane cultivation in high period of its phenology. 


\section{INTRODUCCIÓN}

Según Restrepo Campuzano (2018), Colombia en un país de vocación agropecuaria, donde la agricultura es uno los renglones que aportan altos ingresos al país. Ello se hace cada vez más difícil en el país y los altos costos de producción, costo de agro insumos, costo del agua, valor de las tierras y las políticas cambiantes del estado referente a este sector, dejan menos rentabilidad. De ahí que, las empresas dedicadas a esta actividad se vuelven cada vez más eficientes, adquiriendo tecnologías y adoptando procesos que conlleven a la competitividad.

El sector cañicultor, no es la excepción a dicha situación. Así, como los productores de Caña de Azúcar y los proveedores, han entrado también en esta tónica de la eficiencia, y han empezado a fijarse en los costos de producción, haciendo énfasis en los riegos y más aún, en los estudios físicos del suelo, para luego establecer un verdadero plan de manejo de los suelos y establecer programa de riegos.

Los cañicultores hacen estudios físicos y químicos de sus suelos, pero en términos generales no correlacionan los resultados de los análisis con los programas de riego. Para corregir lo anterior, se puede aprovechar nuevas tecnologías, especialmente los Sistemas de Información Geográfica, que brindan métodos de análisis espacial, permitiendo cuantificar y cualificar propiedades del suelo y llevarlos a un plano, un mapa, como se pretende con el desarrollo de este trabajo de investigación.

Los Sistemas de Información Geográfica permiten además de lo anterior, combinar variables (planta, clima y suelo). Es decir, poseen la capacidad de integrar la información, para obtener resultados más ajustados e integrados, lo cual conlleva a un mejor análisis y recomendaciones reales de acuerdo a la situación.
En la actualidad las actividades agrícolas deben ser eficientes en el manejo de recursos, es así como las geos tecnologías surgen como alternativa a utilizar.

\section{MATERIALES Y METODOS}

La Finca Churimal se encuentra en la vereda $\mathrm{El}$ Hobo a cinco kilómetros de la cabecera del municipio de Roldanillo situada dentro del área plana en las planicies aluviales y de inundación del rio Cauca a una altura de 950 m.s.n.m. Según estimaciones de Dosman, Suárez y Sánchez (2015), los valores climáticos para la finca Churimal son: temperatura media anual de $24^{\circ} \mathrm{C}$, humedad relativa del $80 \%$ y con precipitaciones anuales de $1.100 \mathrm{~mm}$. La finca Churimal cuenta con 93.65 hectáreas sembradas en la variedad de Caña de Azúcar CC8592 (Dosman et al., 2015). La unidad productiva recibe la asistencia técnica del ingenio Riopaila-Castilla, empresa con la que se tiene la modalidad de contrato de compraventa de caña. Se optó por esta variedad dados los tipos de suelo arcillosos y zonas agroecológicas óptimas en donde se adapta muy bien. Se tuvo en cuenta esta variedad por la productividad, la resistencia, las limitaciones edafoclimáticas y al ataque de plagas y enfermedades, así como por la buena vocación para su cosecha mecanizada en zonas agroecológicas optimas; tiene una producción de 130 toneladas por hectárea y un rendimiento de $11.91 \%$ de sacarosa.

La finca Churimal cuenta con un estudio detallado de suelos, clasificación taxonómica, clasificación por capacidad de uso y conformación de grupos homogéneos. Se cartografiaron las siguientes unidades de suelo, las cuales se nombran:

1. Consociación ballesteros $(\mathrm{BL})$

2. Consociación selva (LS) 


\section{Consociación Ricaurte (RT)}

4. Consociación Palmeras (PM)

\section{Consociación catorce $(\mathrm{CA})$}

la hacienda Churimal tiene una extensión de 100 hectáreas en el cultivo de Caña de Azúcar (Saccharum officinarum L). Las coordenadas se toman sobre los linderos del lote No 1 con el objetivo primero de conocer la forma.

Figura 1: Distribución lote suerte No. 1 Hacienda Churimal.

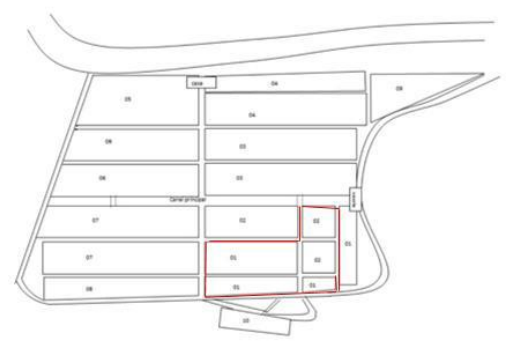

En el lote suerte No 1 demarcado en línea roja en la figura, se estudió la variabilidad espacial de las siguientes propiedades físicas: textura, densidad aparente, densidad real y porosidad a 20 y 40 cms de profundidad, utilizando una cuadrícula con espaciamiento de $50 \mathrm{mts}$ X $50 \mathrm{mts}$ aproximadamente, para un total de 45 puntos de muestreo (Vásquez Polo, Baena Garcia y Menjivar Flores, 2010). Una vez definido el área de estudio, en cada punto, centro de cada cuadricula se toma con GPS las coordenadas, tal como se representa en la figura.

Figura 2: Coordenadas zona de estudio

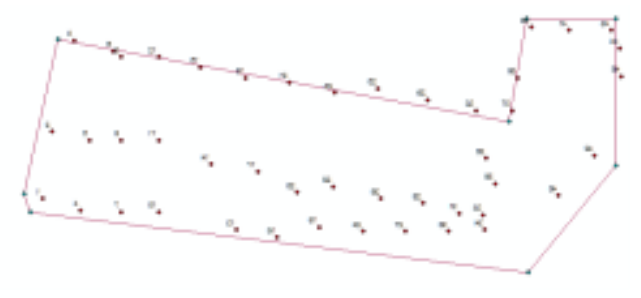

Toma de muestras de suelo y métodos de laboratorio
La precisión con que se deben tomar las muestras obedece a una metodología que permite obtener muestras de buena calidad y que al ser procesadas en laboratorio proporcionen resultados reales, para así determinar texturas de suelos, densidad aparente, densidad real y porosidad, a $20 \mathrm{cms}$ y a $40 \mathrm{cms}$ de profundidad del suelo.

Para la obtención de datos de las propiedades físicas medidas en la suerte 01 de la finca Churimal en la cual se encuentra plantado el cultivo de Caña de Azúcar, se utilizaron

los correspondientes métodos efectuados en el laboratorio de la granja Cedeagro, los resultados se comparan con los valores teóricos que presentan la literatura científica para este tipo de propiedades:

Densidad aparente: método del cilindro.

En campo se hace un hueco con un palin, del cual se extraen dos anillos, uno a $20 \mathrm{~cm}$ y el otro a $40 \mathrm{~cm}$ de profundidad, se lleva a laboratorio y las muestras se secan en un horno por un intervalo de 24 horas a $105^{\circ} \mathrm{C}$. Posteriormente se pesa el anillo más suelo seco y se anota el peso obtenido, después se extrae el suelo del anillo, se pesa el anillo, se obtiene el peso del anillo vacío y se deduce el que tiene el suelo seco (Madriñan Molina, 2009).

Es un buen índice del grado de compactación. En general, los valores de densidad aparente en forma aislada solo deben utilizarse para seguir la evolución de la compactación en un mismo suelo (Gutiérrez, 2014).

\section{Densidad $=$ Masa $/$ Volumen}

Los simples valores de densidad aparente no permiten comparaciones de unos suelos con otros, pues los suelos con texturas más livianas suelen dar valores más altos. Pueden considerarse altos aquellos superiores a 1.3 en suelos de textura fina (arcillosos o franco arcillosos), a 1.4 en suelos de textura media 
(francos a franco limosos), y a 1.6 en suelos de textura gruesa (franco arenosos) (Gutiérrez, 2014).

\section{Densidad real: método del picnómetro.}

Basándose en Madriñan Molina (2009), se procedió a las siguientes etapas de análisis. Para evaluar la densidad real, se pesa el picnómetro y se rotura con el número de la muestra, se anota en la agenda y luego se pesan 5 gramos de suelo en una caja de Petri antes tarada y se depositan el picnómetro el cual se llena con agua destilada hasta $100 \mathrm{ml}$, se pone en la estufa eléctrica a hervir durante 10 minutos y se agita el picnómetro constantemente buscando que la mayoría de poros del suelo sean saturados por el agua, se bajan de la estufa y se dejan reposar de una a dos horas, luego se le agrega más agua a una medida la cual no es constante sino que es determinada por el encargado de realizar las pruebas, se marca con una línea la medida del agua y se pesa, los valores obtenidos se consignan en la agenda como peso de suelo + agua + picnómetro. Se lavan muy bien los picnómetros con abundante agua, se dejan escurrir unos 10 minutos y después se ponen en el horno a $100^{\circ} \mathrm{C}$ durante una hora, se dejan reposar tiempo prudente y se llenan con agua hasta la línea antes marcada y se consignan los datos en la agenda como peso del picnómetro + agua.

\section{Textura.}

Se mide por el método de Bouyoucos. En campo se efectúa un hueco y se retiran dos bolsas cada una de ellas con un kilo de suelo, a 20 y $40 \mathrm{~cm}$ respectivamente. Se procede a llevar las muestras al laboratorio y se ponen a secar durante una semana a temperatura ambiente en bandejas de icopor, posteriormente se procede a realizar el tamizado de cada muestra, las partículas de diámetro menor a $2 \mathrm{~mm}$ son empacadas en una bolsa roturada con la identificación respectiva de cada muestra. Se pesa en una caja de Petri antes tarada 40 gramos de suelo. Se depositan los 40 gramos en un vaso medidor, al cual se le agregan $200 \mathrm{ml}$ de agua y $50 \mathrm{ml}$ de solución calgón. Se deja en reposo durante 10 minutos y después se procede a licuar la solución durante otros 10 minutos. Se deposita el contenido del licuado en una probeta y se llena hasta $1000 \mathrm{ml}$. Se agita durante un minuto y se toma la temperatura. Paso seguido, se introduce el hidrómetro y se registra el valor que este marca a las 2 horas se repite el procedimiento y se anotan los valores en la agenda de trabajo. Por medio de los datos obtenidos y al remplazar la fórmula a continuación se obtiene la clasificación textural del suelo donde se sacó la muestra (Madriñan Molina, 2009).

Ejemplo de remplazo en la formula

Lectura del hidrómetro 26

Factor de corrección 2.41

$$
\begin{aligned}
& 3+2.41=5.41 \\
& \% \text { arena }=100-\underline{5.41 \times 100}
\end{aligned}
$$

\section{0}

$\%$ arena $=86.47$

Lectura de hidrómetro 0,5

Corrección: 2.41

$0.5+2.41=2.91$

$\%$ arcilla $=\underline{2.91 \times 100}$

$\%$ arcilla $=7.27$

$\%$ limo $=100-(\%$ arena $\%$ arcilla $)$

$\% \operatorname{limo}=6.26$

El análisis geoespacial permitió visualizar y graficar la distribución espacial de la variabilidad de las propiedades evaluadas, para dos profundidades de suelo (20 y $40 \mathrm{cms}$ ) (Vásquez Polo et al., 2010). 
Una vez se ha estructurado la base de datos, la información es insertada al programa ArcGIS, mediante las técnicas de interpolación, ya que tal y como indica (Marschallinger, 2009), este es un método sencillo, directo, absolutamente estable y rápido, que permite el cálculo, la estimación 0 predicción del valor de una variable en un lugar donde no se tienen o no se han tomado datos. Es por ello, que la aplicación de este método tiene un aporte significativo, ya que permite determinar las características físicas, como la identificación de los valores: arenas, limos y arcillas, densidad aparente, densidad real y porosidad del suelo. Lo anterior se dio a dos profundidades del suelo, a $20 \mathrm{~cm}$ y a $40 \mathrm{~cm}$ en el mismo punto de muestreo.

Mediante la herramienta de interpolación - IDW (Inverse Distance Weighted), se procedió a generar mapas de las diferentes variables de la parte física de suelos.

Las variables a interpolar son: en profundiades de suelo a 20 y $4 \mathrm{cms}$, son: \% de arena, limo, arcilla, densidad aparente, densidad real $\mathrm{y}$ porosidadad (ver Anexo B).

Para dar respuesta a los objetivos planteados, se sistematizará la información de registros históricos de lluvia. Para así obtener graficas del comportamiento de la distribución de la lluvia y comparar con los meses donde la Caña de Azúcar requiere aplicación de riego.

Se tomó como referencia el desarrollo de la planta de caña de azúcar, específicamente los meses donde presente mayor requerimiento de agua. Es decir, se realizaron comparaciones entre las entradas de agua y el requerimiento de agua en las plantas. Consolidando el histórico de precipitación mensual y anual desde 2011 a 2016, comparando así el nivel agua que entra al suelo vs el requerimiento de la planta durante los meses donde se presenta el periódico critico de riego. La diferencia de láminas se da en milímetros por metro cuadrado y se llevaran a metros cúbicos, que será el volumen de agua aplicar por riego en los meses requeridos para que el cultivo tenga buen desarrollo.

La hacienda Churimal cuenta con 4 pluviómetros, los cuales dan referencia sobre la precipitación y permiten medir la precipitación del área de la hacienda, ya que se registran de manera ordenada y secuencial. Los datos de lluvia aportan información para los proaramas de rieqo.

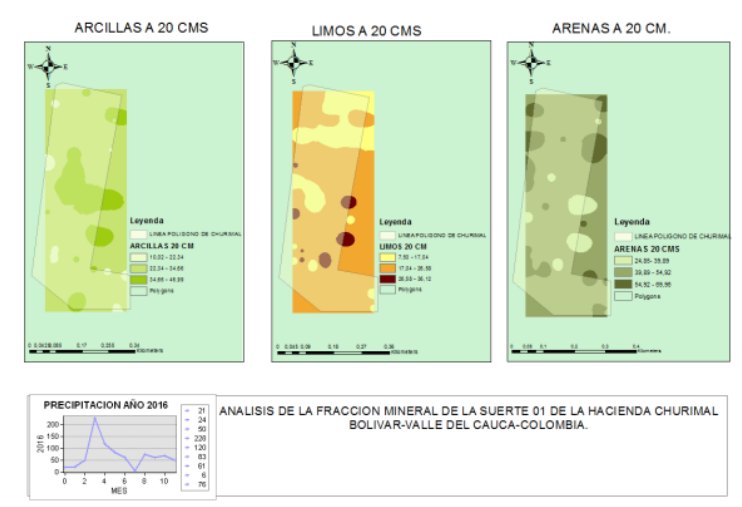

En la imagen podemos encontrar la distribución de arenas, de limos y arcillas, los cuales componen la fracción mineral del suelo. La proporción de las tres fracciones permite obtener la textura, presentando un patrón de distribución irregular. Así mismo se derivan otras variables como lo son la densidad aparente, la densidad real y la porosidad del suelo.

En los mapas se presenta la distribución espacial del porcentaje de arenas, limo y arcilla (se identifican tres rangos: alto, medio y baja presencia). Las fracciones predominantes en áreas corresponden a:

El $47.98 \%$ del área muestreado tiene predominancia de arenas, el $21.67 \%$ del área muestreada tiene predominación de limos y $28.28 \%$ del área muestreada presenta arcillas, que llevado al triangulo textural se obtiene textura a Franco Arcillosa Arenosa. 
Figura 4: Mapa distribución de Arcillas, Limos y Arenas $40 \mathrm{~cm}$ profundidad

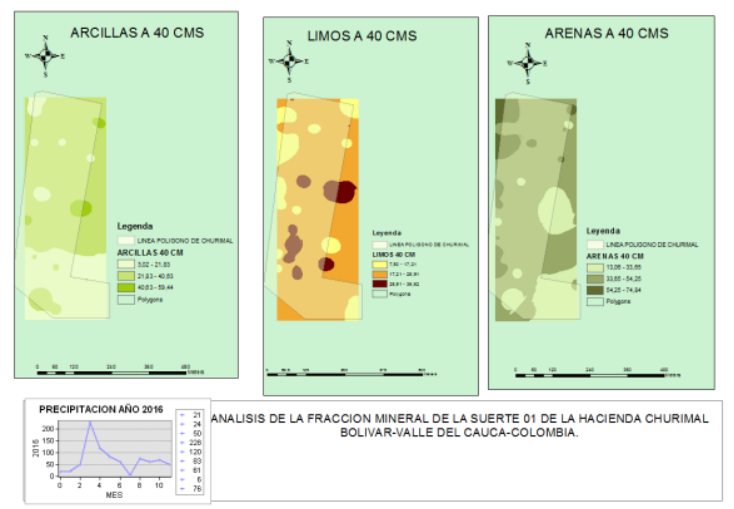

En la figura se presenta mapa de arenas, de limos y arcillas, con patrón de distribución irregular en la suerte No 1 y la combinación de estos tres elementos da origen a la textura. Lo que permite predecir cómo podría ser el movimiento del agua en el suelo.

Además, presentan la distribución de la fracción mineral en toda el área de estudio. En los mapas para contenido de arenas, limo y arcilla (se identifican tres rangos: alto, media y baja presencia) y llevado al triangulo textural se obtiene textura Franco -Arcillosa-Arenosa.

En las dos profundidades (20 y $40 \mathrm{~cm}$ ), coinciden en textura Franco -Arcillosa-Arenosa.

Figura 5: Densidad Aparente a 20 y $40 \mathrm{~cm}$ de profundidad.

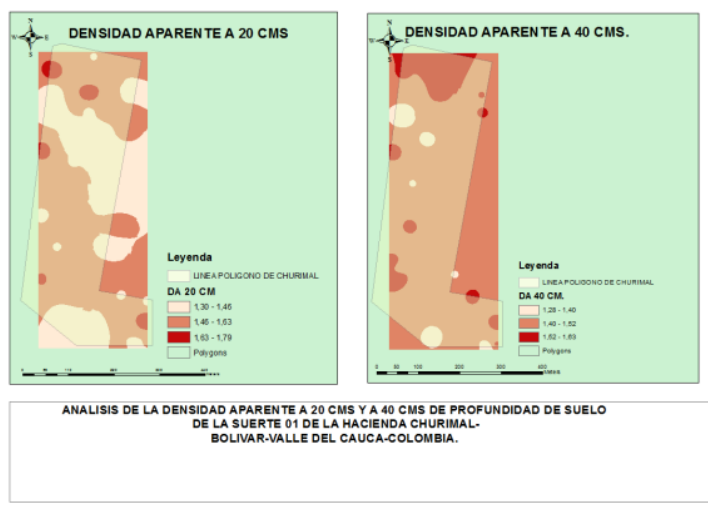

El mapa de densidad aparente a $20 \mathrm{cms}$ de profundidad presenta valores entre $1.30 \mathrm{grs} / \mathrm{cc}$ y $1.79 \mathrm{grs} / \mathrm{cc}$, los cuales coinciden con las zonas de alto y medio contenido de arena.

Hay 5 sitios bien demarcados, con densidades aparentes, entre $1.63 \mathrm{grs} / \mathrm{cc}$ y $1.79 \mathrm{gr} / \mathrm{cc}$ que coinciden con fracciones de arena entre $39.19 \%$ y $69.96 \%$, las cuales presentan densidades aparentes entre $1.30 \mathrm{grs} / \mathrm{cc}$ y $1.63 \mathrm{grs} / \mathrm{cc}$.

En cuanto a la distribución de la densidad aparente a $20 \mathrm{~cm}$, en la suerte No 1, se aprecia una distribución irregular, es decir, no presenta patrones específicos en el área de estudio, siendo los valores que oscilan entre $1.30 \mathrm{gr} / \mathrm{cc}$ y $1.63 \mathrm{gr} / \mathrm{cc}$, los más predominantes.

Como se mencionó anteriormente, los sitios referenciados en color rojo, con densidades que oscilan entre $1.63 \mathrm{gr} / \mathrm{cc}$ y $1.75 \mathrm{gr} / \mathrm{cc}$, presentan una condición diferente en aproximadamente un $10 \%$ del área en estudio.

Por otra parte, en el mapa de densidad aparente a $40 \mathrm{~cm}$ de profundidad presenta valores entre $1.28 \mathrm{grs} / \mathrm{cc}$ y $1.63 \mathrm{grs} / \mathrm{cc}$, los cuales coinciden con las zonas de alto y medio contenido de arena. Hay 9 sitios bien demarcados, con densidades aparentes, entre $1.52 \mathrm{grs} / \mathrm{cc}$ y 1.63 $\mathrm{gr} / \mathrm{cc}$ que coinciden con fracciones de arena entre $33.65 \%$ y $74.84 \%$, las cuales presentan densidades aparentes entre $1.28 \mathrm{grs} / \mathrm{cc}$ y 1.52 grs/cc.

Para la distribución de la densidad aparente a 40 $\mathrm{cm}$, en la suerte No1, se aprecia un comportamiento diferente respecto a los $20 \mathrm{~cm}$ de profundidad del suelo, siendo la densidad que oscila entre $1.40 \mathrm{gr} / \mathrm{cc}$ y $1.52 \mathrm{gr} / \mathrm{cc}$, en un $80 \%$ del área total $y$ en un $15 \%$ se incrementa la porosidad aparente que oscila entre $1.52 \mathrm{gr} / \mathrm{cc}$ y $1.63 \mathrm{gr} / \mathrm{cc}$ demarcada por color rojo oscuro y en menor área 5\% aproximadamente, la densidad que oscila entre $1.28 \mathrm{gr} / \mathrm{cc}$ y $1.40 \mathrm{gr} / \mathrm{cc}$. 
Finalmente, no se aprecian diferencias significativas en las medias de las densidades (a $20 \mathrm{cms}$ y $40 \mathrm{cms}$ ).

Figura 6: Densidad Real a 20 y $40 \mathrm{~cm}$ profundidad

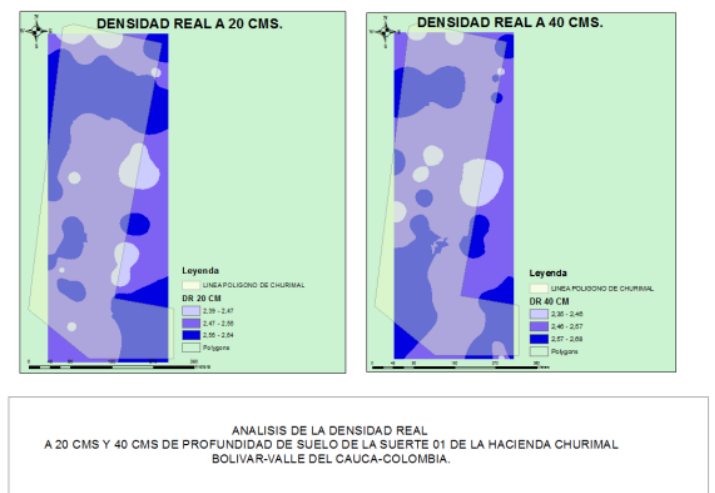

La densidad real a 20 y a $40 \mathrm{cms}$ de profundidad, presentan distribución de manera irregular en la suerte No 1, los valores presentados a $20 \mathrm{~cm}$ de profundidad de $2.35 \mathrm{gr} / \mathrm{cc}$ a $2.64 \mathrm{gr} / \mathrm{cc}$ corresponde con la Textura Franco-Arcillosa, igualmente, a los $40 \mathrm{~cm}$ de profundidad, valores entre $2.35 \mathrm{gr} / \mathrm{cc}$ a $2.68 \mathrm{gr} / \mathrm{cc}$, siendo, los valores de densidad media para las dos profundidades la predominante. Los anteriores valores, se encuentran cercanos a $2.65 \mathrm{gr} / \mathrm{cc}$, cuyos valores se podrían considerar para los suelos de origen mineral.

Figura 7: Porosidad a 20 y $40 \mathrm{~cm}$ de profundidad.

El mapa de porosidad a $20 \mathrm{cms}$ de profundidad, presenta valores entre 33.00 y $51.99 \%$, los cuales coinciden con las zonas de densidad aparente a $20 \mathrm{cms}$ que oscilan entre $1.30 \mathrm{grs} / \mathrm{cc}$ y $1.79 \mathrm{grs} / \mathrm{cc}$,

El mapa de porosidad a $40 \mathrm{cms}$ de profundidad presenta valores entre $32.00 \%$ y $48.99 \%$, los cuales coinciden con las zonas de densidad aparente a $40 \mathrm{cms}$ que oscilan entre $1.28 \mathrm{grs} / \mathrm{cc}$ y $1.63 \mathrm{grs} / \mathrm{cc}$,
Análisis porosidad del suelo y stress hídrico de

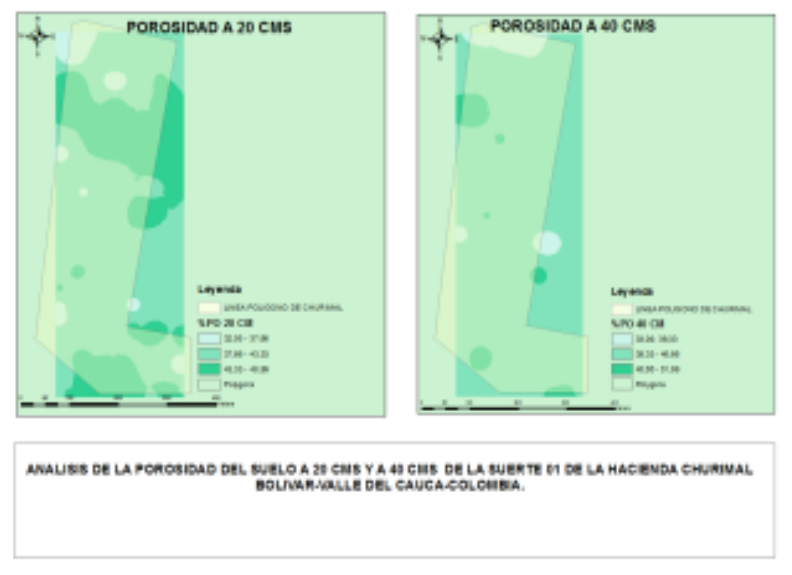

las plantas de caña de azúcar:

Las partículas del suelo, arenas, limos y arcillas, se agrupan formando agregados dentro de los cuales existen espacios vacíos que almacena el agua y los gases. De este modo, el suelo puede considerase como un reservorio de donde las plantas toman el agua necesaria para los procesos de transpiración y para el transporte de los nutrientes del suelo a los tejidos. Por lo tanto, la capacidad de almacenamiento de agua en el suelo varía con la textura y estructura del suelo.

En el caso de las muestras de suelo que se tomaron en la Finca El Churimal, la textura predomínate en la suerte No 1 es Franco Arcillosa Arenosa, que presenta como se mencionó anteriormente almacenamiento y disponibilidad de manera intermedia. Por su parte, la distribución de la precipitación desde los años 2011 a 2016, marca un déficit de lluvias que comparadas con la fenología de la caña de azúcar, muestra los meses donde la caña requiere de agua, por la tanto y aunque la zona cuenta con suelos que ofrecen aceptable retención de agua, lo que se logra aportar a la planta es menor de lo que ésta necesita, de allí la importancia de realizar el adecuado suministro de agua mediante riego, esto con la finalidad de evitar que la planta entre en stress hídrico.

\section{CONCLUSIÓN}


Se identificaron y cuantificaron las propiedades físicas del suelo mediante el siguiente procedimiento: cuadricular en campo, geo referenciar puntos, toma de muestras de suelo, métodos de laboratorio y tabular y sistematizar los datos. Como resultados se obtuvo: texturas, densidad aparente, densidad real y porosidad a los $20 \mathrm{cms}$ y $40 \mathrm{cms}$ de profundidad de suelo.

La variable textura no mostró diferencia entre sus medias, en las dos profundidades de suelo, resultando texturas Franco-Arcillosa-Arenosas; suelo óptimo para el desarrollo de las actividades agrícolas (cultivo de Caña de Azúcar). Pero, cuando se va más allá, analizando las otras variables físicas, (densidad aparente y porosidad), se aprecia que el suelo presenta tendencia a problemas de adensamiento 0 compactación de suelo, lo cual empieza a ser una limitante para el manejo del suelo.

La tendencia al adensamiento del suelo, ocasiona un desarrollo lento en la raíz de la planta y posible atrofiamiento de las raíces, esto se reflejará en que la planta no podrá absorber de manera adecuada los elementos nutritivos, además de que el movimiento y distribución del agua en suelo no será en forma adecuada, ocasionando posiblemente encharcamiento en el suelo.

Se generaron mapas, con información in situ, donde se aporta una visión detallada generalizada del estado de suelo con elementos de juicio para optimizar los recursos (agua, mano de obra, fertilizantes, horas maquina) y así dar un manejo sostenible y rentable en el tiempo.

Las condiciones físicas del suelo van muy relacionadas con la disponibilidad del recurso agua para la planta. De ahí, que se deben tener en cuenta varios factores: uno de ellos es el seguimiento hídrico de la planta, que va muy ligado al desarrollo fenológico, las condiciones climáticas de la zona, especialmente la precipitación y la evaporación. De manera que, al conjugar los elementos, resulten unos volúmenes y épocas donde se programe el riego para así suplir la necesidad, como ocurre en cultivo de Caña de Azúcar ya que la necesidad hídrica se presenta entre los meses 6 y 10 y de no aplicar agua en este periodo de tiempo, disminuirá la producción de manera significativa.

La recopilación de información y la toma de registros de las diferentes actividades que involucren las unidades productivas en el transcurrir de los años es importante para obtener información histórica que permita hacer tabulaciones gráficas y aporten elementos de juicios para la toma de decisiones (mapas, estandarización de procesos, volúmenes de agua, horas máquina, cantidad de fertilizantes). Lo que resultará en una herramienta útil para los procesos administrativos, pues las actividades agrícolas deben ser eficientes para optimizar recursos y obtener márgenes de utilidad en la agro empresa.

El cultivo de Caña de Azúcar, en el Valle del Cauca presenta un área considerable y salvo los ingenios azucareros que cuentan con técnicas y tecnologías de información que se aprovecha para sus cultivares, existen una buena cantidad de proveedores medianos y pequeños con los cuales sería importantes empezar a caracterizar sus suelos en cuanto a las propiedades físicas y químicas, para que tengan elementos de juicio para un mejor manejo del suelo y se refleje en su conservación.

Tecnologías y técnicas de información que ya se encuentran disponibles, son de fundamental importancia en la optimación de los recursos.

\section{REFERENCIAS}

Amaya Estévez, Á., Cock, J. H., Hernández, A. d., y Irvine, J. E. (1995). Biología. En Á. Amaya Estévez, El cultivo de la caña en la zona azucarera de Colombia (págs. 31-62). Santiago de Cali: CENICAÑA. 
ArcGIS Pro. (2018). Comparar métodos de interpolación. Conceptos del conjunto de herramientas Interpolación de ráster. Recuperado el 4 de octubre de 2018, de: https://pro.arcgis.com/es/pro-app/toolreference/3d-analyst/comparing-interpolationmethods.htm

Buzai, G. D., y Baxendale, C. A. (2010). Análisis espacial con sistema de información geográfica. Aportes de la geografía para la elaboración del dianóstico en el ordenamiento territorial. Revista Postgrados UNAH, I(4), 55-67.

Cabrera Barona, P. (2016). Lección 5: Variografía e Interpolación. Salzburg: UNIGIS.

Cañada Torrecilla, R. (2014). Técnicas de interpolación espacial deterministas y exactas media ponderada por el inverso de la distancia y funciones de base radial (Segunda ed.). Madrid: Sistemas y análisis de la información geográfica: Manual de autoaprendizaje con ARC Gis.

Cifuentes Carvajal, A. (2016). Evaluación de diferentes métodos de interpolación para la variable precipitación en el Departamento de Caldas - Colombia. Universidad de Manizales, Facultad de Ciencias e Ingeniería. Manizales: Especialización en Sistemas de Información Geográfica.

CVC, Corporación Autónoma Regional del Valle del Cauca. (2017). Grupo de Recursos Hídricos. Recuperado el 14 de agosto de 2017, de: https://www.cvc.gov.co/cvc/RecursoHidrico/aplic ativos/Climatologia/imagenes/isohietasAnuales/ Valle/cuencasAnual/cuencasAnualAmpliadas/an ualAmpliado.jpg

Chartuni Mantovani, E., y Magdalena, C. (2014). Manual de Agricultura de Precisión. Brasil: Programa Cooperativo para el Desarrollo Tecnológico Agroalimentario y Agroindustrial del Cono Sur.

Choque Tarqui, C. E. (2018). Modelación espacial para la evaluación de la fertilidad del suelo, Estación Experimental Sapecho. Revista de la Carrera de Ingeniería Agrónomica - USMA, 4(2), 1244-1249.

Dosman, A., Suárez, J., y Sánchez, C. A. (2015). Análisis de siete cortes sucesivos del cultivo de caña de azúcar (Saccharum officinarum), para un mejoramiento continuo en labores, costos y productividad, en la finca Churimal vereda el Hobo, Roldanillo, Valle del Cauca. Trabajo de Investigación, Instituto de Educación Técnica Profesional de Roldanillo, Valle - INTEP, Unidad de Ciencias Ambientales y Agropecuarias, Roldanillo.

Dossman, A. (9 de agosto de 2017). Descripción de la finca El Churimal. (J. C. Díaz Serna, Entrevistador)

Duque Franco, A. (2017). Evaluación de las labores agronómicas y variables fenológicas de la variedad de caña de azúcar (Saccharum officinarum) cc 96-2282 en la hacienda Riopaila municipio de Zarzal - Valle Del Cauca. Trabajo de Grado, Instituto de Educación Técnica Profesional de Roldanillo, Valle - INTEP, Unidad de Ciencias Ambientales y Agropecuarias, Roldanillo.

Google Earth. (2014). Vista satelital área rural Roldanillo - Bolívar. Roldanillo - Bolívar, Valle del Cauca, Colombia.

Gutiérrez, K. (2014). Densidad aparente y densidad real del suelo - edafología. Recuperado el 16 de febrero de 2018, de: https://es.scribd.com/document/333992351/Den sidad-aparente-y-densidad-real-del-sueloedafologia

Jímenez Chisica, R. D., y Montes Quintero, O. H. (2015). Implementación de un sistema de información geográfica para el almacenamiento, despliegue y análisis de información sismológica en el observatorio vulcanológico y sismológico de Manizales. Universidad de Manizales, Facultad de Ciencias e Ingeniería, Manizalez. 
Marschallinger, R. (2009). Spatial Stadidistics. Geographical Information

Mosquera G, C. A. (2011). Mapas de productividad como herramienta para entender y gerenciar el cultivo de caña de azúcar. Red Agricultura de Precisión. Recuperado el 24 de enero de 2018, de: http://www.agriculturadeprecision.org/articulos/m onitores-rendimiento/Puntos-Clave-MapaRendimiento-Datos-Confiables_copia(1).asp

QGIS. (2016). Interpolación espacial en detalle. Recuperado el 22 de septiembre de 2018, de: https://docs.qgis.org/2.8/es/docs/gentle_gis_intr oduction/spatial_analysis_interpolation.html

Ramos Rodríguez, H. F. (2012). Factores que afectan la producción-clima-planta-hombre y su incidencia en los sistemnas de producción agrícolas. Palmira: Universidad Nacional de Colombia.

Restrepo Campuzano, A. (2018). Una aproximación al problema de la productividad en el campo colombiano. Bogotá D.C.: Universidad EAFIT.

Riopaila Castilla S.A. (2018). Agricultura de precisión. Procesos productivos. Recuperado el 16 de enero de 2018, de: https://www.riopailacastilla.com/procesos-productivos/

Rodríguez Esteban, J. A. (2014). Exportar vistas y composiciones cartográficas (Segunda ed.). Madrid: Sistemas y análisis de la información geográfica: Manual de autoaprendizaje con ARC Gis.

Suárez A, F. (2011). Propiedades físicas del suelo. Recuperado el 17 de junio de 2018, de: https://es.slideshare.net/cesarfrancisco_77/propi edades-fisica-2

Vásquez Polo, J. R., Baena Garcia, D., y Menjivar Flores, J. C. (2010). Variabilidad espacial de propiedades físicas y químicas en suelos de la granja experimental de la Universidad del
Magdalena (Santa Marta, Colombia). Acta Agronómica, 59(4), 449-4. 